\title{
miR-21, miR-29c, and miR-155 as biomarker to develop minimally-invasive diagnostic in hepatocellular carcinoma patient
}

\author{
P. Lestari ${ }^{1 *}$, N. Qoriansas ${ }^{2}$, D.S. Tanjung ${ }^{1}$, M.S. Fitria1, A.I. Kartika ${ }^{1}$, T. Wardana ${ }^{1}$, \\ N. Ratnasari ${ }^{3}$, S.M. Harjana ${ }^{4}$, T. Aryandono ${ }^{4}$ \\ ${ }^{1}$ Graduate School of Biotechnology, Universitas Gadjah Mada, Yogyakarta, ${ }^{2}$ Graduate \\ Program in Biomedical Sciences, Faculty of Medicine, Universitas Gadjah Mada, \\ Yogyakarta, ${ }^{3}$ Departement of Internal Medicine, RSUP. Dr. Sardjito, Yogyakarta, \\ ${ }^{4}$ Faculty of Medicine, Universitas Gadjah Mada, Yogyakarta
}

DOI: http://dx.doi.org/10.19106/JMedScieSup0048042016019

\section{ABSTRACT}

Hepatocellular carcinoma (HCC) is one of the most prevalent malignancies and the third leading cause of cancer-related deaths worldwide. HCC carries poor prognosis, its incidence and mortality increase annually. The most common hypothesis explaining the problem is the cell's high tendency to metastasize and recur, even after surgical treatment. Therefore, it is important to find new serological biomarkers to detect HCC in early stages. Plasma microRNAs are being actively investigated as minimallyinvasive biomarkers in HCC as well as other human cancers.

This study is aimed to investigate the level of expression of miR-21, miR-29c, and miR-155 as novel serological biomarkers for hepatocellular carcinoma.

This study is preliminary quasi experimental study, and further sample collection is still underway. This study involved 8 HCC patients and 8 healthy controls. Blood sample of HCC patients were obtained from RSUP dr. Sardjito, Yogyakarta, and the patients were selected according to specific inclusion and exclusion criteria. The collected blood samples were treated as follows: plasma isolation, RNA total isolation, cDNA synthesis, quantification by qRT-PCR, data analysis with Biorad CFX ManagerTM Softwere to determine $\mathrm{Cq}$, followed by the calculation of expression levels using Livax Methods.

The result revealed that miR-21 and miR-155 were upregulated, 1.68 fold and 2.38 fold respectively, compared to healthy control. Secondly, miR-29c was downregulated $(3,45$ fold) compared to healthy control.

Based on the result of preliminary study, we concluded that miR-21 acts as oncomiR, while miR-29c and miR-155 act as tumor supressor miR in HCC. The three microRNAs might be detected in $\mathrm{HCC}$ and might be used as minimally-invasive biomarkers in HCC detection.

Keywords: HCC, miR-21, miR-29c, miR-155, minimal invasive, biomarker 